\title{
Magnetism in Coaxial Palladium Nanowires
}

\author{
D. A. Stewart* \\ Cornell Nanoscale Science and Technology Facility, Ithaca, NY 14853
}

(Dated: November 10, 2006)

\begin{abstract}
While bulk palladium is non-magnetic, several recent studies have shown that magnetism can occur in hcp Pd films, fcc twinned Pd nanoparticles, and Pd atomic strands. These studies show that small changes in $\mathrm{Pd}$ atomic configurations can induce magnetic properties. This raises the question of whether nanoscale Pd structures in general are magnetic. In this work, we examine coaxial palladium nanowires in an effort to determine the most stable configurations and their magnetic properties. Relaxed nanowire structures are found using density functional calculations in the plane wave basis. In several metallic systems such as gold, coaxial nanowires have proven to be the lowest energy configurations for ultrathin $(\leq 1 \mathrm{~nm})$ nanowires. We consider magnetism in these structures as a function of diameter and coaxial configuration. These results are also compared to fcc and hcp Pd nanowires of comparable diameters. We find that the $(6,0)$ coaxial nanowire provides the most stable structure and exhibits a magnetic moment of $0.278 \mu \mathrm{B} /$ atom.
\end{abstract}

\section{INTRODUCTION}

The onset of magnetism in palladium crystal structures by lattice distortion or other means has fascinated researchers for over twenty years. In the bulk fcc form, palladium rests on the edge of magnetism with a density of states at the Fermi energy just below the Stoner criterion for ferromagnetism. Past research has shown that a $5 \%$ expansion of the fcc lattice is sufficient to induce magnetism in the fcc crystalline $\mathrm{Pd}^{1-3}$ with a magnetic moment of $0.31-0.35 \mu_{B}$ /atom. In addition, palladium films grown on special substrates take on a hexagonal closedpack crystal structure and possess a finite magnetic moment $\left(0.16 \mu_{B}\right.$ /atom $)$ at the optimum lattice constants ${ }^{4}$. With the advent of fabrication techniques that can produce nanoscale structures such as clusters and nanowires, recent research has focused on how reduced coordination can alter the density of states in $\mathrm{Pd}$ and induce a ferromagnetic transition. Experimental work showed that palladium clusters with a mean radius below $70 \AA$ possess a magnetic moment on the order of $0.2 \mu_{B} /$ atom $^{5}$. Recent theoretical work has linked this magnetic transition in $\mathrm{Pd}$ clusters to changes in symmetry as the cluster transitions from a fcc lattice to a icosahedral multiply twinned particle structure ${ }^{6}$. In addition, palladium plays an integral role in a number of new nanoscale devices such as hydrogen sensors based on $\mathrm{Pd}$ nanowires ${ }^{7}$ and carbon nanotube field effect transistors. Palladium contacts have also been found to provide ohmic contacts for nanotube devices ${ }^{8}$. With recent predictions of novel magnetic forms of carbon, the magnetic character of nanoscale contacts needs to be understood before the magnetic properties of a device can fully be assessed.

Recently Delin et al. examined infinite monowires of $\mathrm{Pd}$ atoms using density functional approaches and found that the equilibrium configuration has a relatively high magnetic moment of $0.7 \mu_{B} /$ atom $^{9}$. Though reduced, measurable magnetic moments were still predicted for short $\mathrm{Pd}$ atom chains between $\mathrm{Pd}$ contacts. By contrast, they found that coaxial $(6,0) \mathrm{Pd}$ nanowires were nonmagnetic. They argued that the $1 \mathrm{D}$ character of the $\mathrm{Pd}$ monowire leads to a Hund's rule magnetism and that this should disappear or become smaller as the number of nearest neighbors increases.

In this study, we investigate a series of coaxial palladium nanowires and explore how magnetism varies with nanowire diameter. Coaxial nanowires are an important group to consider because this form has been observed in thinning experiments for other metallic systems such as gold nanowires ${ }^{10}$. In addition, we examine fcc and hcp nanowires of comparable diameter. These two configurations are particularly interesting because they represent two bulk forms of palladium that are respectively nonmagnetic and magnetic. Since the magnetism in the bulk hcp Pd is due to itinerant electrons, then based on Delin et al. work, there should be a transition from itinerant magnetism to one based on Hund's rule.

\section{APPROACH}

Total energy calculations and structural relaxations were done using the plane wave density functional package Quantum Espresso ${ }^{11}$. Since bulk palladium has such a high magnetic susceptibility, it is crucial that the approach predicts the correct non-magnetic ground state for bulk fcc palladium. Several varieties of local density approximation (LDA) and generalized gradient approximation (GGA) pseudopotentials were considered in this study including ultrasoft Perdew-BurkeErnzerhof (PBE) pseudopotentials ${ }^{12}$ as well as PerdewZunger (LDA) ultrasoft pseudopotentials ${ }^{13}$. In both cases, the effect of including semi-core $d$ states was also considered. However, the LDA pseudopotential with non-linear core correction and a semi-core $d$ state was the only one capable of reproducing the correct nonmagnetic fcc $\mathrm{Pd}$ bulk state at the equilibrium lattice constant. This pseudopotential is used throughout this study for all relevant calculations on palladium nanowire systems. Other research groups using different first principle approaches ${ }^{14,15}$ has also recently noted the inability of GGA Pd pseudopotentials to provide the correct non- 
magnetic fcc ground state.

General coaxial nanowires $(M, N)$ are formed by taking a [111] sheet of palladium and folding it according to vectors defined in the [111] plane (see Kondo and Takayanagi ${ }^{10}$ for full details). Coaxial nanowires can be envisioned as M closely-packed atomic strands wrapped around the wire axis where the angle of the strand with the wire axis ranges from $\theta=0^{\circ}(N=M / 2)$ to $\theta=30^{\circ}$ $(N=0)$. In this study we will consider several coaxial nanowires of the $(M, 0)$ family. The general unit cell for these nanowires consists of $2 M+2$ atoms. The $(M, 0)$ nanowires can also be thought of as two rings of $\mathrm{M}$ atoms each where the atoms in the second ring are rotated by $2 \pi / M$ relative to the first ring. An atom also sits at the center of the nanowire between each ring. Figure 1 provides both head-on and side views of $(5,0)$ and $(6,0)$ coaxial nanowires. The side view of the $(6,0)$ nanowire shows the closely packed atomic strands wrapping around the wire axis. The head-on view for both nanowires shows the central atom as well as the first $M$ atom ring below.

For the nanowire calculations, periodic supercells were considered where the cell length in the $x$ and $y$ directions perpendicular to the wire is given by $10.58 \AA$. The initial cell size in the $z$ direction was determined by the particular coaxial nanowire structure. For each system, the supercell and atomic positions were allowed to relax to their minimum energy configuration. For all the calculations discussed in this paper, we use a 34 Ry energy cut-off for wavefunctions and a 340 Ry cut-off for the charge density. Brillouin zone sampling is done over an unshifted [1x1x20] Monkhurst-Pack grid.

\section{RESULTS}

The total energy as a function of nanowire diameter was examined for $(M, 0)$ coaxial nanowires where $\mathrm{M}$ ranges from 4 to 9 . The energy per atom is given for a number of structures in Figure 1. The energy per atom for the $\mathrm{Pd}$ atom, atomic strand, and bulk fcc palladium are also indicated in Figure 1. The results indicate that all the coaxial nanowires except for the $(4,0)$ case are more stable than the palladium atomic strand. Out of the coaxial nanowires considered, the $(6,0)$ nanowire provides the most stable structure. However, it should be noted that the energy difference between many of the coaxial nanowires is fairly small. This is similar to carbon nanotubes where many chiral configurations are roughly equivalent in terms of energy. This indicates that it would be possible to observe several coaxial nanowire configurations during palladium nanowire fabrication. Small fcc and hcp palladium nanowires were also considered in this study and possess slightly higher energy configurations than the $(6,0)$ coaxial nanowire.

The magnetic moment as a function of nanowire diameter is shown in Figure 2 for both the coaxial nanowires and the fcc and hcp nanowires. All of the coaxial

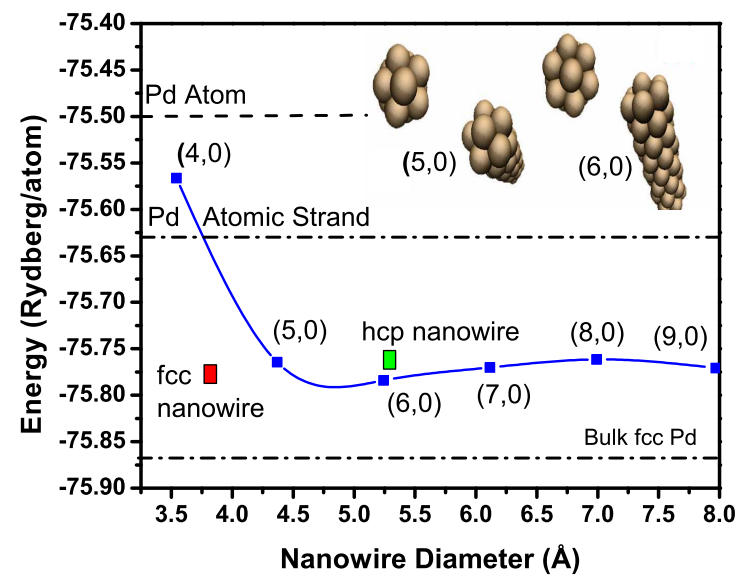

FIG. 1: (color online) The energy per atom for a series of coaxial palladium wires is shown for a range of diameters. The total energy for single unit cell fcc and hcp palladium nanowires is also presented. Dashed lines indicate the calculated energies for a free palladium atom, a palladium atomic strand, and bulk fcc palladium. Head-on and size views of $(5,0)$ and $(6,0)$ coaxial nanowires are shown in the upper right hand corner of the figure.

nanowires relaxed to a magnetic ground state during the calculations. It is interesting to note that although bulk fcc palladium is non-magnetic, the single cell fcc palladium nanowire possesses a small magnetic moment $(0.065 \mu \mathrm{B} /$ atom $)$. The magnetic moment for the $(4,0)$ nanowire is also small $(0.07 \mu \mathrm{B} /$ atom $)$. This is most likely due to the close proximity of palladium atoms which encourages overlap between $d$ orbitals reducing the magnetic moment. However, the $(5,0)$ nanowire displays the highest magnetic moment $(0.5 \mu \mathrm{B} /$ atom $)$ of the coaxial nanowires considered. In fact, the magnetic moment of the $(5,0)$ nanowire is greater than that observed in hexagonal close-packed films of palladium. The $(6,0)$ nanowire exhibits a magnetic moment of $(0.278$ $\mu \mathrm{B}$ /atom) and the other nanowires with larger diameters exhibit similar magnetic moments. The calculated energy difference between possible magnetic and nonmagnetic ground states for $(6,0)$ palladium nanowires is small $(\approx 3 \mathrm{meV})$, indicating that magnetism in these nanowires would be observed primarily at low temperature. The magnetic moment calculated for the palladium atomic strand $(0.725 \mu \mathrm{B} /$ atom $)$ agrees well with the result $(0.7 \mu \mathrm{B} /$ atom $)$ found in the previous work by Delin et $a .^{9}{ }^{9}$. However Delin et al. found that the $(6,0)$ coaxial nanowire did not possess a magnetic moment in contrast to our current work. For their work, they used a full-potential linear muffin tin orbital approach (FPLMTO) and the specific calculation details and whether they performed structural relaxation were not noted. As the previous discussion of pseudopotentials has shown, 


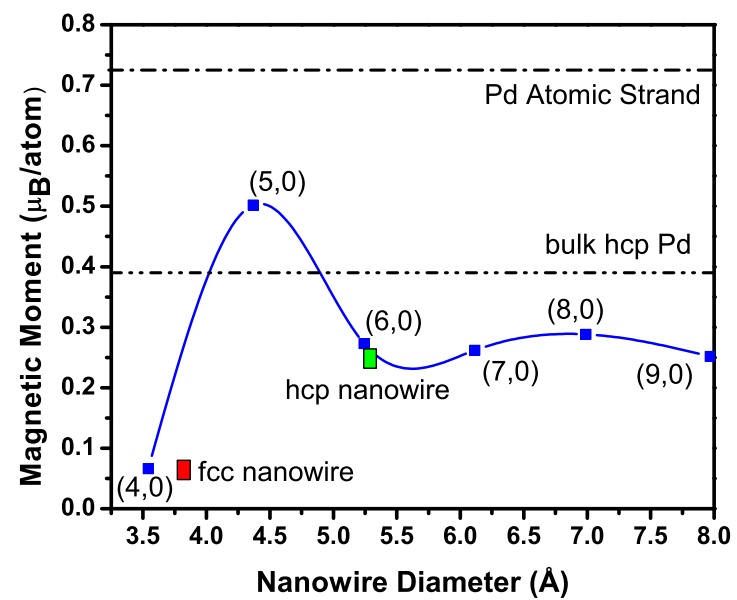

FIG. 2: (color online) The magnetic moment per atom is shown for a series of coaxial palladium wires. The magnetic moment per atom for single unit cell fcc and hcp palladium nanowires is also presented. Dashed lines indicate the calculated magnetic moments for a palladium atomic strand and bulk hcp palladium. Bulk fcc palladium and the palladium atom are both non-magnetic and are not shown on the graph.

bulk palladium truly sits at the edge of magnetism and small differences in predicted energies due to the use of different pseudopotential, basis sets, or calculation resolution can affect magnetic property predictions.

In order to determine if the magnetism in the $(6,0)$ nanowire is localized on the rings of atoms in the system, we also examined an alloy $\mathrm{PdAu}(6,0)$ nanowire where the center atoms are replaced by gold. This system has no magnetic moment, indicating that the central strand of atoms play a role in the observed magnetism. In addition, we also considered a $\mathrm{PdCo}(6,0)$ nanowire where the center atoms are replaced by cobalt. In this case, the average magnetic moment in the system is enhanced to $0.47 \mu \mathrm{B}$ /atom. This enhancement is not surprising due to the high magnetic susceptibility of palladium. In fact, over forty years ago, single magnetic impurities in bulk palladium were shown to induce small magnetic moments in a large number $(\approx 200)$ of the neighboring palladium $\operatorname{atoms}^{16,17}$.

\section{CONCLUSION}

In this work, we have considered the stability and magnetic properties of coaxial palladium nanowires. The $(6,0)$ nanowire provides the lowest energy configuration and possesses a magnetic moment of $0.278 \mu \mathrm{B} /$ atom. Coaxial nanowires in the same chiral family over the diameter range considered are all magnetic. For coaxial nanowires, $(7,0)$ and greater, the magnetic moment and ground state energy is fairly close to that found in the $(6,0)$ nanowire. This indicates that experiments geared towards generating coaxial palladium nanowires will most likely find a range of chiral configurations.

\section{Acknowledgments}

Calculations were perfomed on the Intel Cluster at the Cornell Nanoscale Facility which is part of the National Nanotechnology Infrastructure Network (NNIN) funded by the National Science Foundation (NSF).
* stewart@cnf . cornell.edu

1 L. Fritsche, J. Noffke, and H. Eckardt, J. Phys. F: Met. Phys. 17, 943 (1987).

2 H. Chen, N. E. Brener, and J. Callaway, Phys. Rev. B 40, 1443 (1989).

3 S. S. Alexandre, E. Anglada, J. M. Soler, and F. Yndurain, Phys. Rev. B 74, 054405 (2006).

4 E. Huger and K. Osuch, Europhys. Lett. 63, 90 (2003).

5 T. Taniyama, E. Ohta, and T. Sato, Europhys. Lett. 38, 195 (1997)

6 L. Vitos, B. Johansson, and J. Kollar, Phys. Rev. B 62, R11957 (2000).

7 F. Favier, E. C. Walter, M. P. Zach, T. Benter, and R. M. Penner, Science 293, 2227 (2001).

8 A. Javey, J. Guo, Q. Wang, M. Lundstrom, and H. Dai, Nature 424, 654 (2003).
9 A. Delin, E. Tosatti, and R. Weht, Phys. Rev. Lett. 92, 057201 (2004).

10 Y. Kondo and K. Takayanagi, Science 289, 606 (2000).

11 S. Baroni et. al., http://www.pwscf.org.

12 J. P. Perdew, K. Burke, and M. Eznerhof, Phys. Rev. Lett. 77, 3865 (1996).

13 J. P. Perdew and A. Zunger, Phys. Rev. B 23, 5048 (1981).

14 S. S. Alexandre, M. Mattesini, J. M. Soler, and F. Yndurain, Phys. Rev. Lett. 96, 079701 (2006).

15 T. Kana, D. Legut, M. Sob, unpublished.

16 G. G. Low and T. M. Holden, Proc. Phys. Soc. London 89, 119 (1966).

17 A. Oswald, R. Zeller, and P. H. Dederichs, Phys. Rev. Lett. 56, 1419 (1986). 